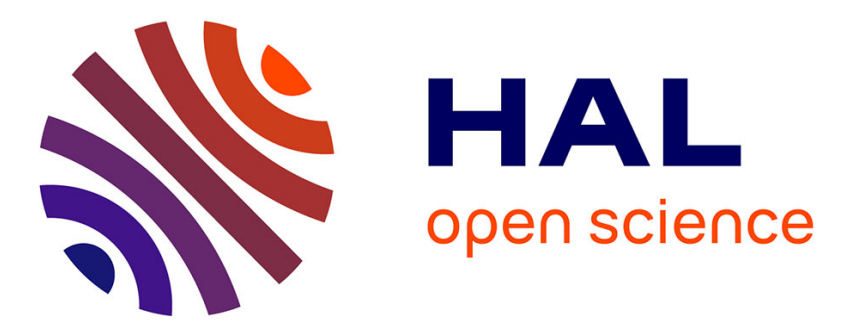

\title{
Electrically tunable optoelastic interaction range of nematic colloids
}

\author{
Luigino Criante, Francesco Bracalente, Liana Lucchetti, Francesco Simoni, \\ Etienne Brasselet
}

\section{- To cite this version:}

Luigino Criante, Francesco Bracalente, Liana Lucchetti, Francesco Simoni, Etienne Brasselet. Electrically tunable optoelastic interaction range of nematic colloids. Soft Matter, 2013, 9 (22), pp.5459-5463. 10.1039/C3SM50273B . hal-00834140

\author{
HAL Id: hal-00834140 \\ https://hal.science/hal-00834140
}

Submitted on 1 Mar 2018

HAL is a multi-disciplinary open access archive for the deposit and dissemination of scientific research documents, whether they are published or not. The documents may come from teaching and research institutions in France or abroad, or from public or private research centers.
L'archive ouverte pluridisciplinaire HAL, est destinée au dépôt et à la diffusion de documents scientifiques de niveau recherche, publiés ou non, émanant des établissements d'enseignement et de recherche français ou étrangers, des laboratoires publics ou privés.

\section{(ㄷ)(1) $\$$}

Distributed under a Creative Commons Attribution - NonCommerciall 4.0 International 


\title{
Electrically tunable optoelastic interaction range of nematic colloids
}

\author{
Luigino Criante, ${ }^{\text {ab }}$ Francesco Bracalente, ${ }^{a}$ Liana Lucchetti, ${ }^{a}$ Francesco Simoni ${ }^{a}$ \\ and Etienne Brasselet ${ }^{\text {cd }}$
}

\begin{abstract}
We report on the electrical tuning of the interaction range between colloidal particles in nematic liquid crystals. The experimental demonstration relies on the study of the dynamics of a real colloidal particle made of a glass sphere in the presence of an artificial laser induced colloid that results from the local distortion of the nematic by a Gaussian light beam. The optoelastic interaction range between such a pair of colloids is shown to be fully controlled electrically. Moreover, all the observations are well described by an analytical model that accounts for the field induced reorientation of the liquid crystal and the overlap between the long range director distortion around both colloids.
\end{abstract}

In materials science, the long-range orientational order of liquid crystal mesophases has been found useful to selfassemble colloidal particles. ${ }^{1}$ Basically, the interaction potential of colloids in liquid crystals is anisotropic ${ }^{2}$ and leads to various self-assembled structures of microparticles. ${ }^{3}$ In particular, natural liquid crystal topological defects are of paramount importance to achieve stable two-dimensional self-assembled structures. ${ }^{4}$ Extension to three-dimensional colloidal architectures has great application potential in optics towards the realization of photonic crystals and metamaterials in the visible domain, for which the so-called blue phases of liquid crystals are predicted to be promising candidates. ${ }^{5}$

In this context, optical strategies enabling controlled distant manipulation of particles or colloidal structures are desirable. This involves light-driven force and torques mediated by the long-range interaction of liquid crystals. This was originally demonstrated almost a decade ago by Musevic and coworkers who unexpectedly trapped a nematic colloid using high numerical aperture laser tweezers ${ }^{6}$ - unexpected since gradient force trapping ${ }^{7}$ is forbidden when the refractive index of the object is lower than that of the surrounding medium. The first explanation distinguished two different regimes: ${ }^{8}$ (i) below the onset of the so-called optical Fréedericksz transition, ${ }^{9}$ the dressed nematic colloid behaves as a high refractive index particle compared to the surrounding medium and is consequently trapped by conventional optical gradient forces;

${ }^{a}$ Dip. di Scienze e Ingegneria della Materia, dell'Ambiente ed Urbanistica and CNISM, Univ. Politecnica delle Marche, 60131 Ancona, Italy

${ }^{b}$ Center for Nano Science and Technology@PoliMi, Istituto Italiano di Tecnologia, Via Giovanni Pascoli, 70/3, 20133 Milano, Italy

'Univ. Bordeaux, Laboratoire Ondes et Matière d'Aquitaine, UMR 5798, F 33400 Talence, France

${ }^{d}$ CNRS, Laboratoire Ondes et Matière d'Aquitaine, UMR 5798, F 33400 Talence, France (ii) above the onset of the transition, the optically reoriented area behaves as a 'ghost' colloid and minimization of the elastic free energy is responsible for trapping, as is the case between two real colloidal particles. ${ }^{2}$ Since then, counterintuitive thresholdless optical reorientation of homeotropic nematic films (i.e. provided with perpendicular alignment boundary conditions) at normal incidence and moderate focusing conditions has been unveiled. ${ }^{\mathbf{1 0}, 11}$ This has led to the recent demonstration of distant optical trapping of nematic colloids even under poorly focused Gaussian beams, ${ }^{12}$ in stark contrast to conventional optical trapping. Besides bulk reordering schemes, surface-driven optical manipulation of particles and complex colloidal structures in liquid crystals has also been proposed recently. ${ }^{13}$ The combination of optoelastic interaction with the action of external electric or magnetic field is expected to bring other degrees of freedom to the existing toolbox for the versatile control of colloids in anisotropic fluids, which we explore in the present work.

Here we report on the electrical tuning of the optoelastic interaction range between real and laser-induced ghost

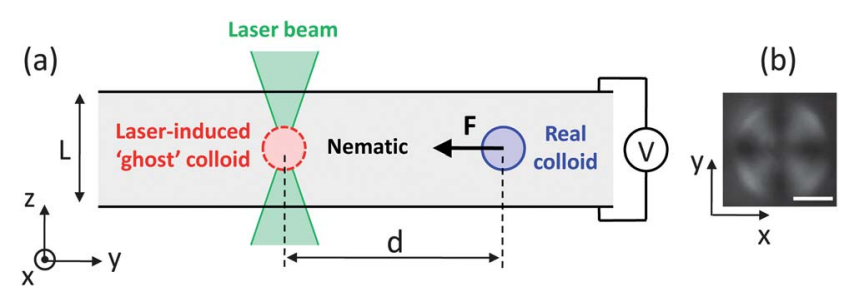

Fig. 1 (a) Experimental sketch. A real colloidal particle in a nematic film interacts with a laser induced 'ghost' colloid and may experience an attracting optoelastic force $F$. (b) White light imaging of the dipolar nematic real colloid between crossed polarizers (along $x$ and $y$ ) where the dark cross refers to the axisymmetric director profile around the particle due to homeotropic boundary conditions both on the particle surface and film substrates. Scale bar is $5 \mu \mathrm{m}$. 
colloidal particles in nematics, see Fig. 1(a). The optoelastic interaction range between such a pair of colloids is experimentally demonstrated to be fully controlled by low-voltage electric fields. In particular, we show that the optoelastic interaction can be electrically screened in the case of a nematic with positive dielectric anisotropy. Moreover, we develop a model that accounts for (i) the electrical realignment of the liquid crystal along the electric field direction and (ii) the overlap between the long-range orientational distortion around both colloids. We stress that our approach is conceptually more intuitive than previous attempts based on the analogy with dipolar and quadrupolar interaction and the extension to materials with negative dielectric anisotropy is also discussed.

\section{Experimental}

The liquid crystal sample is a $L=50 \mu \mathrm{m}$ thick film of a $4^{\prime}-n$ pentyl-4-cyanobiphenyl (5CB) nematic compound sandwiched between two parallel glass substrates provided with ITO electrodes that are transparent in the visible domain. Both substrates are covered by dimethyloctadecyl[3-(trimethoxysilyl) propyl]ammonium chloride (DMOAP, from FLUKA), which ensures strong homeotropic anchoring. Silica spheres (from Bang Laboratories Inc.) with $2.5 \mu \mathrm{m}$ radius and refractive index $n_{\mathrm{s}}=1.37$, also covered by DMOAP, are dispersed in the nematic before capillary filling of the cells. The refractive indices of 5CB along and perpendicular to the director (i.e. a unit vector that defines the local average molecular orientation) are $n_{\|}=1.71$ and $n_{\perp}=1.54$ at the used wavelength. The trapping light source is a linearly polarized Gaussian laser beam at $532 \mathrm{~nm}$ wavelength that is focused at normal incidence onto the sample. The beam divergence angle in the nematic is $\theta_{0}=3.1^{\circ}$, hence preventing gradient force optical trapping as quantitatively demonstrated in previous work. ${ }^{\mathbf{1 2}}$ The real-time dynamics of the real colloid is retrieved from CCD camera video imaging by using a white light illumination from the opposite side with respect to the incident laser beam.

In practice, we select an isolated dipolar colloidal particle, see Fig. 1(b), lying at an altitude $z$ approximately in the middle of the cell. The laser beam is focused at the same altitude, thereby preventing spurious heating effects due to ITO absorption, ${ }^{14}$ at an initial distance $d_{0}$ from the particle. Then, the particle motion is recorded for various values of $d_{0}$ and applied voltage $V$ at $1 \mathrm{kHz}$ frequency, the incident laser beam power being fixed at $P=10 \mathrm{~mW}$. In all cases, the experimental conditions (polarization state direction and location of the laser beam with respect to dipolar colloid) correspond to 'easy-axis' optical trapping, ${ }^{8}$ namely, the colloid trajectory is a straight line.

The typical behavior of the dipolar colloid dynamics is shown in Fig. 2 for different applied voltages when $d_{0}=23 \mu \mathrm{m}$. The larger the $V$ the slower the first stage of the motion though the final stage of the dynamics follows a voltage independent scenario. Noticeably, the colloid is no longer attracted towards the laser beam above a well-defined voltage value $V=V_{\mathrm{q}}$ named the quenching voltage, see curve (e) in Fig. 2. The slowing down of the trapping time $\tau$ as a function of the applied voltage for $d_{0}=17,23,29$ and $35 \mu \mathrm{m}$ is shown in Fig. 3, where dashed lines

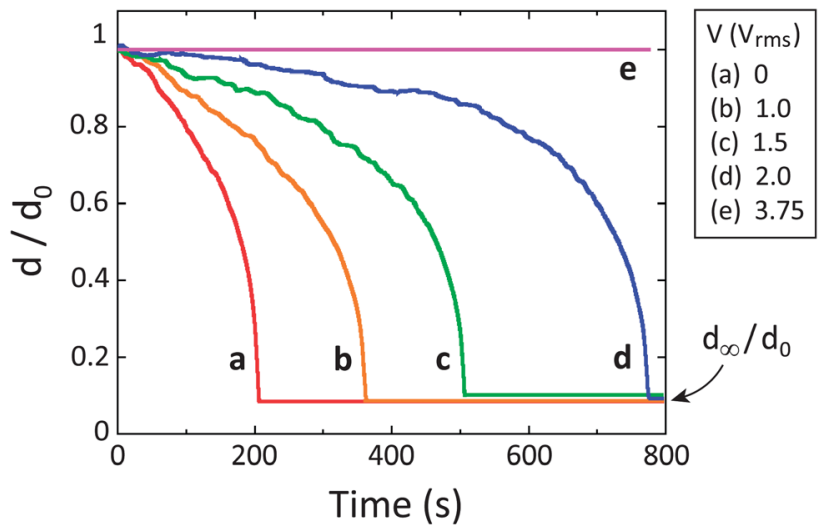

Fig. 2 Distance $d$ between real and ghost colloids as a function of time for different values of the applied voltage $V$ for an initial distance $d_{0}=23 \mu \mathrm{m}$, where $d_{\infty}$ refers to the equilibrium position.

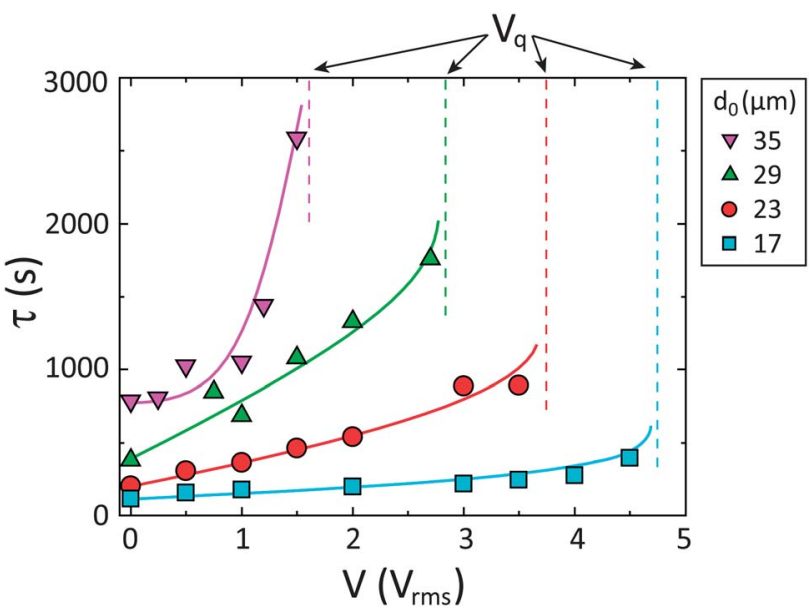

Fig. 3 Time duration $\tau$ of the trapping process versus the applied voltage for different values of initial separation distance $d_{0}$. Solid curves are guide for the eye.

refer to the quenching voltage. Experimentally, the quenching voltage is determined as the one for which the particle keeps its initial position for observations longer than $45 \mathrm{~min}$. This is summarized in Fig. 4, where the area below the curve $V=V_{\mathrm{q}}$ refers to a nonzero attracting force whereas above it the optoelastic force vanishes. All these results indicate that the attractive part of the interaction potential strongly depends on voltage. This can be quantitatively characterized experimentally from the evaluation of the total force $\boldsymbol{F}$ exerted on the colloid at the early stage of the dynamics, just after the laser beam is turned on. Indeed, in that case, $\boldsymbol{F}$ is well approximated by its attracting Coulomb-like contribution ${ }^{\mathbf{8 1 2}}$ when $d_{0}$ is large enough with respect to the equilibrium position $d_{\infty}$.

For this purpose, neglecting the inertia of the glass sphere, $\boldsymbol{F}=F \boldsymbol{y}$ [see Fig. 1 for sign conventions, $\boldsymbol{y}$ being the unit vector along the $y$ axis] is balanced with the viscous drag force $\boldsymbol{F}_{\mathrm{D}}$ exerted on the moving particle by the surrounding fluid. Using the low Reynolds number expression $\boldsymbol{F}_{\mathrm{D}}=6 \pi R \eta(\partial d / \partial t) \boldsymbol{y}$, where $\eta=86 \mathrm{mPa} s$ is the effective viscosity for 5CB in our case ${ }^{15}$ we get $F=6 \pi R \eta(\partial d / \partial t)$. An example is shown in Fig. 5(a) for $d_{0}=29 \mu \mathrm{m}$ and $V=0$. By writing the attracting part of the 


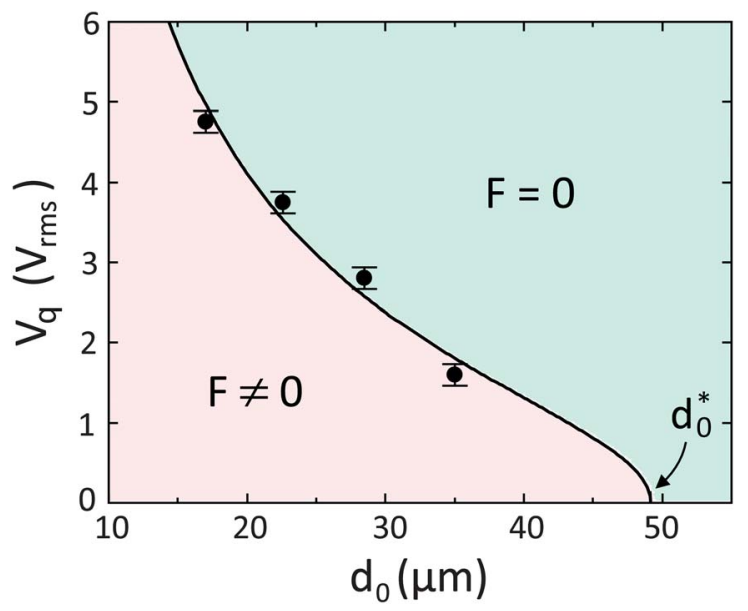

Fig. 4 Quenching voltage $V_{\mathrm{q}}$ as a function of the initial separation distance $d_{0}$. At fixed $d_{0}$, an attracting force is exerted on the particle when $V<V_{\mathrm{q}}(F \neq 0)$ whereas the optoelastic interaction is screened for $V>V_{\mathrm{q}}(F=0)$. The solid curve refers to the model, see eqn (3).
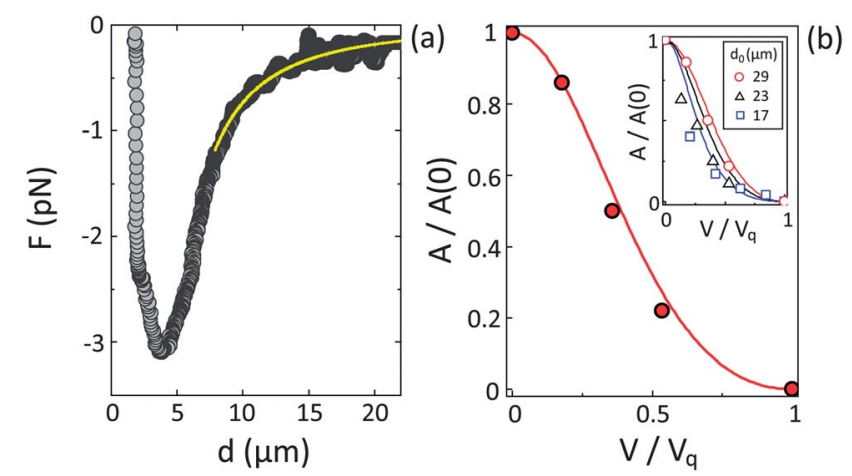

Fig. 5 (a) Trapping force $F$ acting on the particle $v$ s. the distance $d$ to the laser beam axis, at $V=0$. The solid curve refers to the best fit from the attractive part of the total force exerted on the particle, $F_{A}=A / r^{2}$. (b) Dependence of parameter A versus applied voltage for $d_{0}=29 \mu \mathrm{m}$. Markers: experimental data. Solid curve: model, see eqn (4). Inset: confrontation between the experiment and model for three values of initial separation distance $d_{0}$.

total force in the form $\boldsymbol{F}_{\mathrm{A}}=\left(A / r^{2}\right) \boldsymbol{y}$, with $A>0$, it is therefore possible to extract the coefficient $A$ by fitting $F(d)$, which is typically done over the first ten of microns of the particle displacement. This is illustrated in Fig. 5(a) where the solid curve refers to the best fit. It is therefore possible to retrieve the dependence of the attracting force on the applied voltage for various values of $d_{0}$. The result is shown in Fig. 5(b) for $d_{0}=29$ $\mu \mathrm{m}$, the data for other values of $d_{0}$ being given in the inset.

In the next section, we elaborate a model to describe all our observations, thereby unveiling the underlying physical mechanism at work for such an electrically controlled optoelastic interaction range of nematic colloids.

\section{Model}

With the aim at describing the interaction between the real (R) and ghost (G) nematic colloids, a representation of the distorted director field around them is needed. For this purpose, the general method consists of the minimization of the total (elastic + electric + optical) free energy, which accounts for the coupling between the director field and both the light field and the quasistatic electric field. The presence of defects either due to the real colloid or associated with the light-induced ghost colloid is another ingredient to include. Formally, this can be handled numerically. However, to our knowledge, this has not been reported yet and is beyond the scope of the present study. In fact, here we want to exploit the experimentally well-established $1 / r^{2}$ dependence of the attractive part of the force exerted on the real colloid ${ }^{6,8,12}$ to discuss the effects of the applied electric field.

For this purpose, we propose an effective analytical approach by restricting the analysis to a large enough interparticle distance, without intending to describe the director profile neither of the ghost colloid nor around the real one. Therefore, both the presence of defects and the optical reorientation issues are discarded. Moreover, under the assumption of axial symmetry and small reorientation amplitude, we retain only the fundamental mode for the tilt angle $\vartheta$ of the director with the $z$ axis, $\vartheta(r, z)=\theta(r) \sin (\pi z / L)$. According to this, we obtain a differential equation for the reorientation amplitude $\theta(r)$, which results from the minimization of the total (elastic + electric) free energy (see for instance ref. 16, though paying attention that we are dealing here with positive dielectric anisotropy). Consistent with an asymptotic description, we merely keep the linearized version of it, namely

$$
\frac{\mathrm{d}^{2} \theta}{\mathrm{d} r^{2}}+\frac{1}{r} \frac{\mathrm{d} \theta}{\mathrm{d} r} \quad\left[\frac{\pi^{2}}{L^{2}}\left(1+\tilde{V}^{2}\right)+\frac{1}{r^{2}}\right] \theta=0,
$$

where we introduced the reduced voltage $\tilde{V}=V / V_{\mathrm{F}}$ with $V_{\mathrm{F}}=\pi \sqrt{ } K_{3} / \varepsilon_{0} \varepsilon_{\mathrm{a}}, K_{3}$ being the Frank elastic constant for bend deformations and $\varepsilon_{\mathrm{a}}>0$ being the dielectric anisotropy of the relative permittivity of the nematic at the used voltage frequency. Eqn (1) is further simplified assuming $r^{2} \gg \xi^{2}$, where $\xi=(L / \pi)\left(1+\tilde{V}^{2}\right)^{-1 / 2}$ is the electrical coherence length. This gives

$$
\frac{\mathrm{d}^{2} \theta}{\mathrm{d} r^{2}} \quad \frac{\theta}{\xi^{2}}=0,
$$

The solution of eqn (2) is written as $\theta^{(x)}(r)=\theta_{0}^{(x)} \exp (r / \xi)$ with $x=(R, G)$ and $\theta_{0}^{(x)}$ referring to the effective extrapolated tilt value at $r=0$, whose physical meaning will be discussed hereafter. ${ }^{17}$

The basic idea to describe the voltage-induced optoelastic interaction screening relies on the fact that, physically, the attraction force originates from a spontaneous molecular rearrangement that lowers the total free energy of the system. This necessarily implies the mutual influence between the real and the ghost colloid, which occurs when, at some location, both $\theta^{(R)}$ and $\theta^{(G)}$ are larger than a typical value $\theta_{T}$ given by thermal orientational fluctuations. Indeed, one can speak of director reorientation only when the director orientation goes beyond the average fluctuation. The quenching conditions are therefore expressed as $\theta^{(R)}\left(r_{T}^{(R)}\right)=\theta^{(G)}\left(r_{T}^{(G)}\right)=\theta_{T}$ and $d_{0}=r_{T}^{(R)}+r_{T}^{(G)}$. This gives 


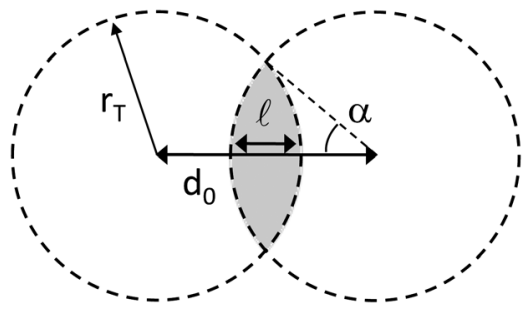

Fig. 6 Sketch of the overlapping reorientation areas of the two colloids with the definition of the overlapping distance $\ell$ and the angle $\alpha$.

$$
\tilde{V}_{\mathrm{q}}=\left[\begin{array}{ll}
\left(d_{0}^{*} / d_{0}\right)^{2} & 1
\end{array}\right]^{1 / 2} \text { with } d_{0} \leq d_{0}^{*}
$$

where $d_{0}^{*}=(L / \pi)\left[\ln \left(\theta_{0}^{(R)} / \theta_{T}\right)+\ln \left(\theta_{0}^{(G)} / \theta_{T}\right)\right]$ is the interparticle distance above which the real and ghost colloids no longer interact at $V=0$. Eqn (3) is compared to experimental data by performing a fit with two adjustable parameters, $d_{0}^{*}$ and $V_{\mathrm{F}}$. As shown by the solid curve in Fig. 4, a fairly good agreement here with best fit values $d_{0}^{*}=49.2 \mu \mathrm{m}$ and $V_{\mathrm{F}}=1.82 V_{\mathrm{rms}}$. Finally, we note that the above assumption $r^{2} \gg \xi^{2}$ can be written in our case $d_{0}^{2} \gg \xi^{2}\left(\tilde{V}_{\mathrm{q}}\right)$, that is to say, from eqn (3), $d_{0}^{* 2} \gg(L / \pi)^{2}$. Since we found $d_{0}^{*} \simeq L$, we conclude that our approach has satisfying self-consistency.

Next, we aim at describing the voltage dependence of parameter $A$ that appears in the expression of $\boldsymbol{F}_{A}$. The starting point is to note that previous work suggested that $A$ scales as the square of the characteristic radius of the laser-induced reorientation area. ${ }^{12}$ The above successful analysis of the quenching voltage, however, suggests the overlap between the reorientation profiles of the real and ghost colloids to be the relevant feature. We therefore propose the scaling $A \propto \ell^{2}$, where $\ell$ is the overlap distance, as depicted in Fig. 6. Assuming for simplicity that $r_{T}^{(R)}=r_{T}^{(G)}=r_{T}$ we get $\ell=2 r_{T}\left(\begin{array}{cc}1 & \cos \alpha\end{array}\right)$ where $\cos$ $\alpha=d_{0} /\left(2 r_{T}\right)$ with $d_{0}<d_{0}^{*}$. Therefore, from $r_{T}=\left(d_{0}^{*} / 2\right)\left(1+\tilde{V}^{2}\right)^{-1 / 2}$ and eqn (3), we get

$$
\frac{A(\tilde{U})}{A(0)}=\frac{1}{1+\tilde{U}^{2} \tilde{V}_{\mathrm{q}}^{2}}\left(\frac{\sqrt{1+\tilde{V}_{\mathrm{q}}^{2}} \sqrt{1+\tilde{U}^{2} \tilde{V}_{\mathrm{q}}^{2}}}{\sqrt{ } 1+\tilde{V}_{\mathrm{q}}^{2} 1}\right)^{2}
$$

where $\tilde{U}=V / V_{\mathrm{q}}$ and $0 \leq \tilde{U} \leq 1$. The confrontation between the model and experimental data is shown in Fig. 5(b) for $d_{0}=29$ $\mu \mathrm{m}$, where the fit shown in Fig. 4 is used to fix the value of $\tilde{V}_{\mathrm{q}}\left(d_{0}\right)$. The obtained agreement is fairly good despite the simplicity of the model, which emphasizes the physical background of our model based on the asymptotic description of the director profiles around the colloids. Consistently, the agreement between the predicted and experimentally measured voltage dependent interaction potential is all the more satisfying when the initial separation distance is large, as shown in the inset of Fig. 5(b).

\section{Discussion}

Importantly, we recall that the observed anisotropic attractive potential, i.e. the 'easy-axis' optical trapping, ${ }^{8}$ actually supports the fact that the observed laser induced distorted area results from the optical torque density exerted on the director field rather than heating effects. It is indeed possible to distinguish thermal effects from purely dielectric optical reorientation effects merely by looking at the trapping area between crossed polarizers. At $U=0$, a bright spot is observed as a consequence of reorientation, which fades away as $U$ is increased (not shown here). We thus conclude that present experiments deal with the nematic mesophase only.

Consequently, we expect that the role of long-range orientational order in liquid crystal mesophases is worth investigating in various configurations of optical micromanipulation of colloids in anisotropic fluids, see for instance the recent review paper by Trivedi et al. ${ }^{18}$ and references therein. Moreover, the ability to fully control the optoelastic interaction of colloids by external electric fields in the case of positive dielectric anisotropy, as presented here, can be extended to the case of materials with negative dielectric anisotropy. Indeed, we anticipate a giant long-range order in that case as a result of the realignment of the director perpendicular to the electric field. In particular, an anti-quenching voltage $V=V_{\overline{\mathrm{q}}}$ can be introduced, above which the interaction starts to take place for a situation with initially screened interaction, i.e. $d_{0} \geq d_{0}^{*}$. Following the reasoning of our model, the following expression can be derived:

$$
\tilde{V}_{\overline{\mathrm{q}}}=\left[\begin{array}{ll}
1 & \left(d_{0}^{*} / d_{0}\right)^{2}
\end{array}\right]^{1 / 2} \text { with } d_{0} \geq d_{0}^{*},
$$

where $V_{\mathrm{F}}$ should be written in that case as $V_{\mathrm{F}}=\pi \sqrt{ } K_{3} / \varepsilon_{0}\left|\varepsilon_{\mathrm{a}}\right|$. This is illustrated in Fig. 7. Interestingly, the interaction range $\Lambda=d_{0}^{*}\left(1 \quad \tilde{V}^{2}\right)^{-1 / 2}$ is found to diverge as $V \rightarrow V_{\mathrm{F}}$, when the whole nematic film is at the onset of the electric Fréedericksz transition threshold. Such a situation should lead to novel hierarchical self-assembling protocols in soft matter that would be worth investigating experimentally.

To conclude, it has been demonstrated experimentally that the optoelastic interaction between a light field and a colloidal particle in nematic liquid crystals can by tuned at will by using an external electric field that controls the distance above which

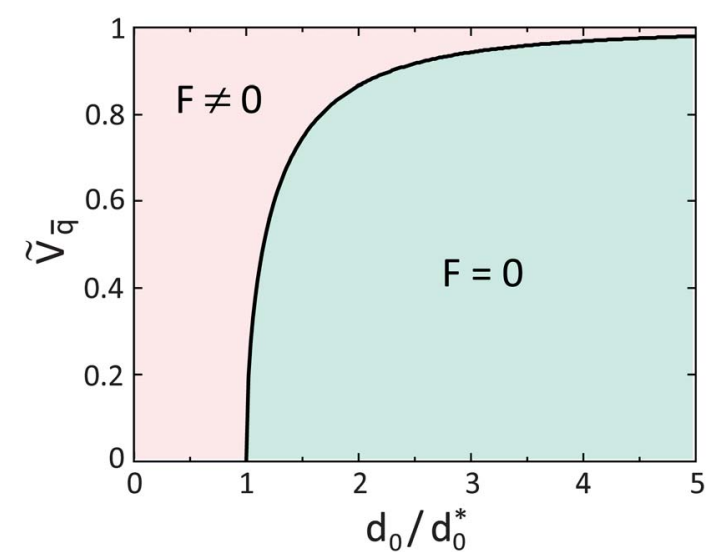

Fig. 7 Anti quenching voltage $\tilde{V}_{\mathrm{q}}$ as a function of the initial interparticle distance for nematic liquid crystals with negative dielectric anisotropy, see eqn (5). 
the interaction is screened by thermal orientational fluctuation of the nematic host. Experimental results are supported by a simple analytical model that is able to handle the case of positive or negative dielectric anisotropy of the liquid crystal. Interaction quenching has been found in the former case whereas anti-quenching and diverging long-range interaction has been predicted in the latter case.

\section{References}

1 P. Poulin, H. Stark, T. C. Lubensky and D. A. Weitz, Science, 1997, 275, 1770.

2 M. Yada, J. Yamamoto and H. Yokoyama, Phys. Rev. Lett., 2004, 92, 185501.

3 M. Ravnik, M. Skarabot, S. Zumer, U. Tkalec, I. Poberaj, D. Babic, N. Osterman and I. Musevic, Phys. Rev. Lett., 2007, 99, 247801.

4 I. Musevic, M. Skarabot, U. Tkalec, M. Ravnik and S. Zumer, Science, 2006, 313, 954.

5 M. Ravnik, G. P. Alexander, J. M. Yeomans and S. Zumer, Proc. Natl. Acad. Sci. U. S. A., 2011, 108, 5188.

6 I. Musevic, M. Skarabot, D. Babic, N. Osterman, I. Poberaj, V. Nazarenko and A. Nych, Phys. Rev. Lett., 2004, 93, 187801.

7 D. G. Grier, Nature, 2003, 424, 810.
8 M. Skarabot, M. Ravnik, D. Babic, N. Osterman, I. Poberaj, S. Zumer, I. Musevic, A. Nych, U. Ognysta and V. Nazarenko, Phys. Rev. E: Stat., Nonlinear, Soft Matter Phys., 2006, 73, 021705.

9 B. Y. Zel'dovich, N. V. Tabiryan and Y. S. Chilingaryan, Sov. Phys. JETP, 1981, 54, 32.

10 E. Brasselet, Opt. Lett., 2009, 34, 3229.

11 E. Brasselet, J. Opt., 2010, 12, 124005.

12 L. Lucchetti, L. Criante, F. Bracalente, F. Aieta and F. Simoni, Phys. Rev. E: Stat., Nonlinear, Soft Matter Phys., 2011, 84, 021702.

13 A. Martinez, H. C. Mireles and I. I. Smalyukh, Proc. Natl. Acad. Sci. U. S. A., 2011, 108, 20891.

14 In practice, melting of the liquid crystal may occur at higher power $(P>20 \mathrm{~mW}$, while we used $P=10 \mathrm{~mW}$ power in the experiments) when a beam is focused near the ITO boundary surface, which is not the case in our experiments.

15 H. Stark and D. Ventzki, Phys. Rev. E: Stat., Nonlinear, Soft Matter Phys., 2001, 64, 031711.

16 A. Rapini, J. Phys., 1973, 34, 629.

17 Note that the amplitudes $\theta^{(R)}$ and $\theta^{(G)}$ originate from the elastic distortion of the particle and the trap, respectively, and have therefore two distinct physical origins.

18 R. P. Trivedi, D. Engstrom and I. I. Smalyukh, J. Opt., 2011, 13, 044001. 